Brit. Heart J., 1965, 27, 105.

\title{
ELECTRODE JELLY IN ELECTROCARDIOGRAPHY
}

\author{
BY \\ DAVID LEWES \\ From the Cardiac Department, Bedford General Hospital
}

Received January 27, 1964

In the early days of electrocardiography it was the recognized practice to place a patient's hands and left foot in three large saline pots. With the introduction of contact electrodes more than fifty years ago (James and Williams, 1910) and their later improvement by Cohn (1920), this ritual of partial immersion slowly gave way to swathing the limbs in bandages incorporating saline pads and malleable metal electrodes. Saline pads, however, brought new difficulties: bedclothes were liable to become moistened with saline in bedridden patients resulting in short-circuiting, and drying of the pads caused increasing skin resistance particularly during prolonged experimental observation. These disadvantages were overcome by Boas (1928) who dispensed with saline pads for the purpose of cardiotachometry. He found that two concave metal chest electrodes of one inch diameter filled with soft green soap as a contact material and tied to the chest by tapes gave consistently satisfactory tracings during three hours of continuous recording. Following this early demonstration that a simple contact medium, consisting of an almost neutral mixture of potassium oleate and glycerine, could give acceptable electrical recordings of the heart beat, it later became fashionable in electrocardiography to use an abrasive electrode jelly or paste rich in sodium chloride. Because of the inherent tendency for string galvanometer machines to "overshoot", Jenks and Graybiel (1935) devised a method for overcoming skin resistance and avoiding "overshoot" by concocting an electrode paste rich in sodium chloride and a pumice abrasive which effectively removed the superficial layers of the skin, thus greatly enhancing skin conductivity (Bell, Knox, and Small, 1939). Commercial electrode jellies and pastes consisting principally of sodium chloride and an abrasive soon became available, so that Russell (1935), using a string galvanometer machine, was able to recommend Cambridge electrode jelly for routine electrocardiography.

In 1939 Bell et al. conducted comparative resistance experiments with a string galvanometer instrument employing as electrode contact material, 1 per cent sodium chloride solution, Cambridge electrode jelly, Jenks' and Graybiel's "Boston Paste", and soft green soap (sapo mollis viridis B.P.). They found no difference in the cardiograms yielded respectively by expensive Cambridge electrode jelly and soft green soap which at that time cost one penny an ounce. These workers were unable to find any earlier report of a systematic investigation into the properties of electrolytes in overcoming skin resistance, apart from the work of Hartridge (1931) which dealt with the effect of grease-removing substances.

Maxwell (1957) considered that special electrode jelly, which he described as messy, inconvenient, and time-consuming to use, was unnecessary for recording electrocardiograms. He found that rubbing the selected areas of skin and the electrodes with a damp sponge gave results as good as commercial electrode jelly, and later he compared the water and jelly methods in 20 cases (Maxwell, 1958). He expressed surprise that the results agreed so well. His original but unconventional ideas, adversely criticized on theoretical grounds by Browne (1957), have been forgotten or ignored, 
so that Maxwell appears to be the only worker who has refused to bow to tradition by his abandoning commercial electrode jelly or other special electrode contact material for routine electrocardiography. Too often in the past has progress in medicine been frustrated by traditional practice being permitted, perhaps even encouraged, to ride roughshod over new ideas and unconventional methods. The virtual neglect of Maxwell's modest but important reports serve as a contemporary reminder of the oppressive influence of the habit-pattern in medicine.

Certain manufacturers of commercial electrode jelly seem as hesitant in 1963 as they were in 1939 (Bell et al.) to divulge the composition of their products. Nevertheless, it seems likely that there has been little alteration over the years of the electrolyte content of commercial jelly, since a recent analysis of one widely used preparation shows a sodium chloride content of approximately 13 per cent (C. R. Watts, 1963, personal communication).

When assessing the efficiency of an electrode contact material it should be remembered that electrode jellies and pastes containing abrasives and a high concentration of sodium chloride were originally devised for use with relatively insensitive string galvanometer instruments of ultra-low input impedance. Valve electrocardiographs, now almost universally used for routine electrocardiography, have an input impedance at least 100 times that of the string, so that high body resistance is not so disadvantageous when using modern machines as it was with the earlier instruments (Bell et al., 1939). However, when using string instruments and older amplifying valve electrocardiographs of low input impedance, such as the early Cambridge Electrite, commercial electrode jelly remains the electrode contact material of choice (L. Goulding, 1964, personal communication; E. G. Short, 1964, personal communication).

It occurred to me that the principle that commercial electrode jelly is still essential for electrocardiography when using modern machines of high input impedance might be based more upon tradition than upon experiment, and that the high sodium chloride and abrasive content of these preparations might be unnecessary for recording acceptable electrocardiograms with modern equipment.

The purpose of this paper is to record an investigation employing high input impedance instruments, which compared the cardiogram obtained when using an electrode contact material rich in sodium chloride and abrasive with that recorded when an inexpensive chloride-free and abrasivefree lubricating jelly containing only small amounts of inorganic ions was used, to study the cardiogram obtained when dry electrodes were applied to unprepared skin, and to inquire into the limitations, if any, of Maxwell's (1957) water method.

\section{THE INVESTIGATION}

More than two years ago it was found that electrode jelly of high sodium chloride content was causing corrosion of chest electrodes. A change to Johnson and Johnson's K-Y Jelly, an innocuous preparation widely used as a lubricant for internal examinations, was made experimentally because it did not corrode metal instruments and because its cost was substantially less than commercial electrode jelly. The K-Y Jelly consistently gave satisfactory electrocardiograms, so that for the past two years commercial electrode jelly has been abandoned in favour of K-Y lubricant which has been used for recording over 4000 electrocardiograms in this Hospital's Cardiac Department and wards. These records were taken with a doublechannel and two single-channel Cardiopan electrocardiographs of 4 and $10 \mathrm{M}$ ohms input impedance, respectively. Moreover, when the Elmquist Junior portable machine of $2 \mathrm{M}$ ohms input impedance at $50 \mathrm{c} / \mathrm{s}$ was used in 200 patients during the course of domiciliary and private work, K-Y Jelly regularly gave cardiograms as acceptable as those yielded by commercial electrode jelly or by the Cardiopan instruments using K-Y Jelly.

Chemical Composition and Manufacture of Johnson and Johnson's $K-Y$ Jelly. The gel system consists of cellulose gums and alginates and the addition of glycerine and glycols confers lubricant properties. Approximately 1 per cent of the preparation consists of a preservative system, and a chemical agent, which completes the sterilization of the jelly in the sealed tube, is hydrolysed to a harmless material.

The jelly is chloride-free but potassium ions, added in the neutralization process, adjust the $p \mathrm{H}$ to approximately 5.5 units. Chloride would be present as an impurity in the ingredients making up the gel. 
TABLE

Comparison of some Properties of K-Y Jelly (Johnson and Johnson), Lubafax (Burroughs Wellcome), and CAMBridge Electrode Jelly

\begin{tabular}{l|c|c|c|c}
\hline Preparation & Chloride content & $\begin{array}{c}\text { Specific conductivity } \\
(\mathrm{mhos} \text { per ml. at } \\
\left.23^{\circ} \mathrm{C} .\right)\end{array}$ & $\begin{array}{c}\text { Comparative resistance } \\
\left(\text { ohms at 22 }{ }^{\circ} \mathrm{C} \text {. }\right)\end{array}$ & $p \mathrm{H}$ \\
\hline K-Y Jelly & Negligible & $0.01555-0.01749$ & 2150 & 5.8 \\
Lubafax & Negligible & 0.00233 & 9750 & 4.2 \\
Cambridge & $8 \%$ & 0.09720 & 1550 & 5.5 \\
N/10 K Cl & - & 0.01239 & - & - \\
\hline
\end{tabular}

Electrolytes present derive from boric acid, approximately 1 per cent sodium and potassium ions (approximately 0.3 per cent). The preparation is abrasive-free.

Degree of Resistance. The specific conductivity, resistance, and other properties of K-Y Jelly, Lubafax (Burroughs Wellcome), a tragacanth and glycerine jelly containing 0.2 per cent potassium hydroxyquinoline, and Cambridge electrode jelly are compared in the Table.

The apparatus used was a Mullard Conductivity Cell and Bridge and an Avometer to measure the resistance.

Comparative Electrocardiographic Tests. The apparatus consisted of a battery-powered Elmquist Junior portable electrocardiograph of $2 \mathrm{M}$ ohms input impedance at $50 \mathrm{c} / \mathrm{s}$ employing standard Elmquist nickel silver on brass limb electrodes each of approximately $15 \mathrm{sq} . \mathrm{cm}$. contact area, and a shallow all-metal circular suction chest electrode of $2.6 \mathrm{~cm}$. external diameter.

Four healthy female subjects aged 10, 14, 27, and 82 years respectively were studied. Since external electrical interference, dirty electrodes, careless application of electrodes, and faulty standardization are important causes of unsatisfactory and especially low-voltage tracings, attention was directed to their avoidance in this study: the apparatus was earthed and observations were made as far removed from 50cycle interference as possible and at times when this was likely to be minimal. New electrodes were used. Test preparations, gently rubbed into the skin with the finger, avoiding excess, were smeared thinly onto the electrodes, which were snugly positioned on the prepared skin then firmly but not tightly secured by perforated bands. The apparatus was standardized $(1 \mathrm{~cm} . / 1 \mathrm{mV})$ and the standard included with the subject in the circuit in each lead of every preparation tested.

Lubafax, K-Y, and a commercial electrode jelly were tested seriatim in order to reduce the risk of contamination of skin, hands, and apparatus with sodium chloride contained in the commercial electrode jelly. After testing each preparation electrodes and rubber bands were dried after scrubbing in running water, and the chest in the position of V5 was thoroughly washed and dried with sterile gauze. Fresh skin areas on the limbs were used for each preparation tested.

Serial electrocardiograms using Lubafax, K-Y Jelly, and commercial electrode jelly were equally acceptable both as regards the height and form of the deflections and maintenance of full standardization with the subject in the circuit (Fig. 1).

Though previous experience had repeatedly shown that K-Y Jelly yielded an electrocardiogram the equal of that obtained with commercial electrode jelly, a comparable performance in a critical experiment by Lubafax with a comparative resistance exceeding four times that of K-Y and of more than six times that of commercial electrode jelly, was unexpected. A comparable performance by K-Y and Lubafax, pitted against their respective specific conductivity, suggested that the combination of a large electrode area, thin film, and low current, requires but little assistance in the form of added electrolytes to lower skin resistance sufficiently for the recording of a satisfactory electrocardiogram when using modern equipment of high input impedance. This reasoning further suggested that any aqueous proprietary preparation marketed in a tube might serve as an efficient electrode jelly. This proved to be so with Cetavlex Paste (I.C.I.) which was tested solely because it was to hand when the other preparations were being examined (Fig. 1).

Culinary and Toilet Preparations used as Electrode Contact Material. The author purchased from a self-service store the first six tubed preparations found on the shelves, namely: hand cream (Evan Williams), toothpaste (Gibbs), mayonnaise, marrons glacés, French mustard, and tomato paste. Each of these products tested against commercial electrode jelly (lead II) yielded an electrocardiogram as acceptable as the 


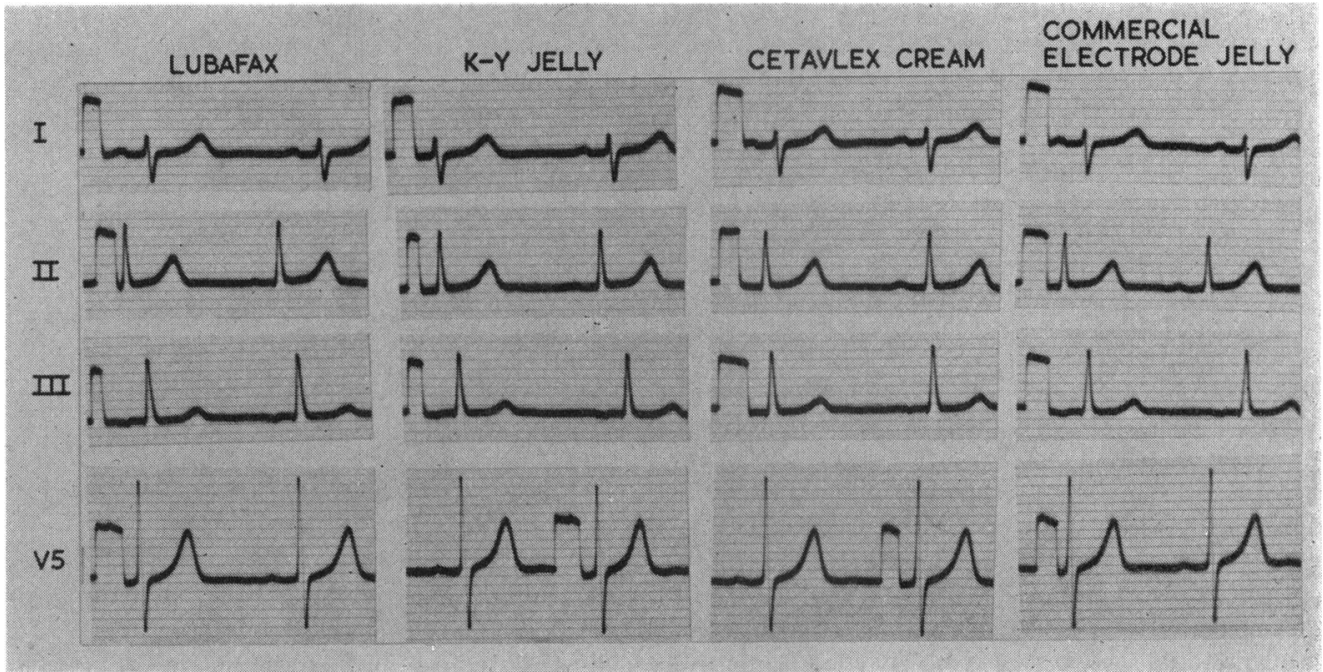

Fig. 1.-Serial electrocardiograms recorded from a girl aged 10 years using as electrode contact material: Lubafax (Burroughs Wellcome), K-Y Jelly (Johnson and Johnson), Cetavlex cream (I.C.I.), and commercial electrode jelly.

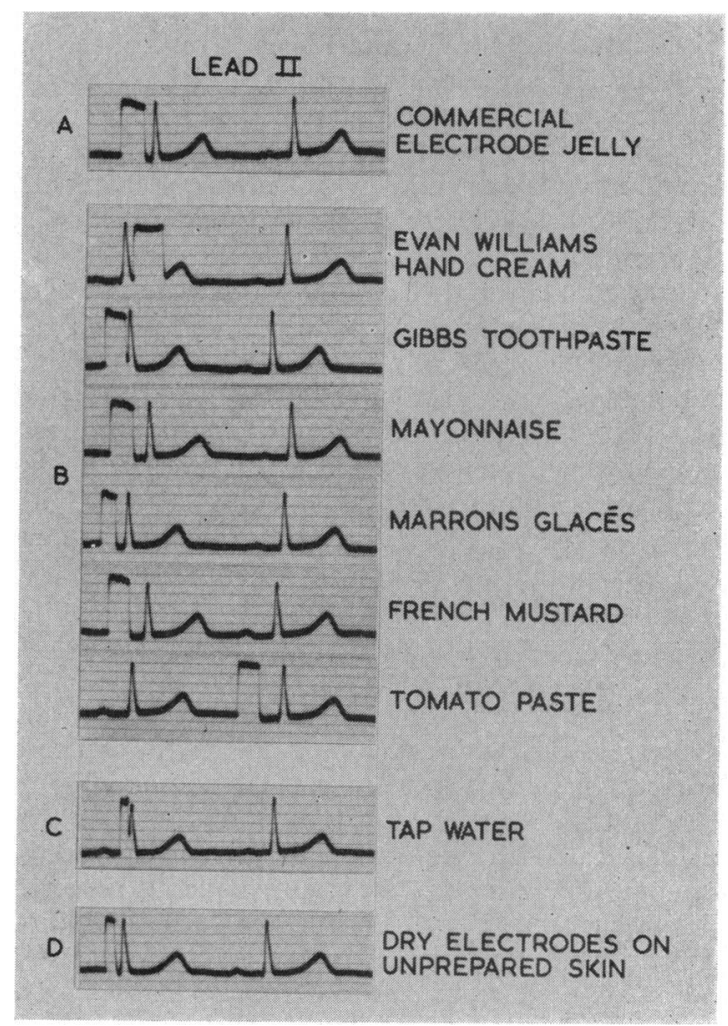

Fig.2.-Serial electrocardiograms (lead II) of a healthy girl aged 10 years using as electrode contact material: (A) commercial electrode jelly; (B) six culinary and toilet preparations; (C) tap waler; (D) dry polished electrodes on unprepared skin for 15 minutes. 
control both as regards the height of the complexes and the maintenance of standardization (Fig. 2). The hand cream proved to be the best: it was pleasant, relatively inexpensive, clean, and more convenient to use than K-Y Jelly, since it could be rubbed into the skin without trace after recording the electrocardiogram. Williams' hand cream, subsequently used for a trial period for routine electrocardiography in the Cardiac Department, gave results as satisfactory as K-Y or commercial electrode jelly.

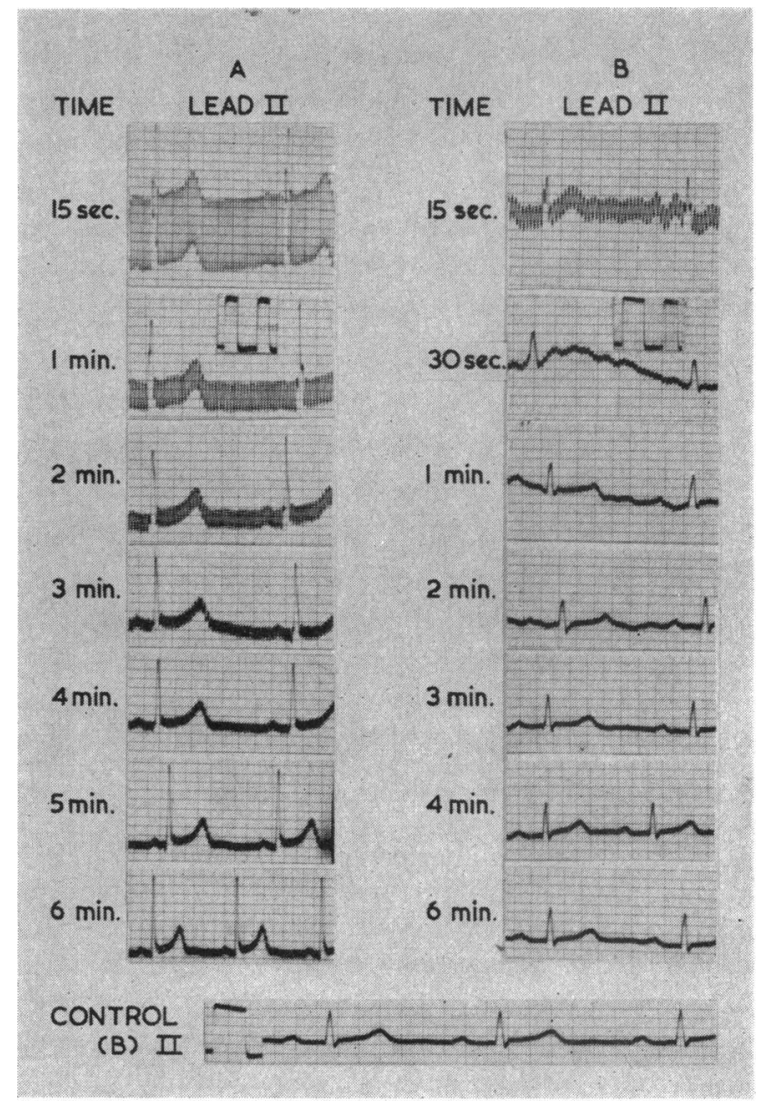

FIG. 3.-Electrocardiograms recorded at timed intervals after applying dry polished electrodes to unprepared skin.

Direct Electrode-Skin Contact. J.L., a healthy 14-year-old girl with a natural unmoistened skin, was connected to an Elmquist Junior electrocardiograph by new limb electrodes which had been washed with distilled water, dried, and polished with sterile cotton wool before being applied to the natural skin. Serial tracings of lead II recorded at timed intervals immediately after applying the electrodes show gross interference at 15 seconds, but progressive improvement in subsequent tracings. After six minutes a tracing free from interference was recorded (Fig. 3). The experiment was repeatable when the washed dried electrodes were applied to fresh areas of skin. At the end of this experiment the sodium chloride content of the skin underlying an electrode area of $15 \mathrm{sq} . \mathrm{cm}$. was estimated as approximately $6 \mathrm{mg}$. The skin was thoroughly cleaned with sterile cotton wool moistened with distilled water, and its chloride content was measured against an equal quantity of control cotton wool after elution of the cotton wool with distilled water. In Y.C., aged 27, comparable results were obtained. Allowing for variation in the speed of recording, the tracings at 4 and 6 minutes are indistinguishable from the control record using commercial electrode jelly (Fig. 3).

The experiment was then modified by placing one drop of distilled water on the unprepared skin before 


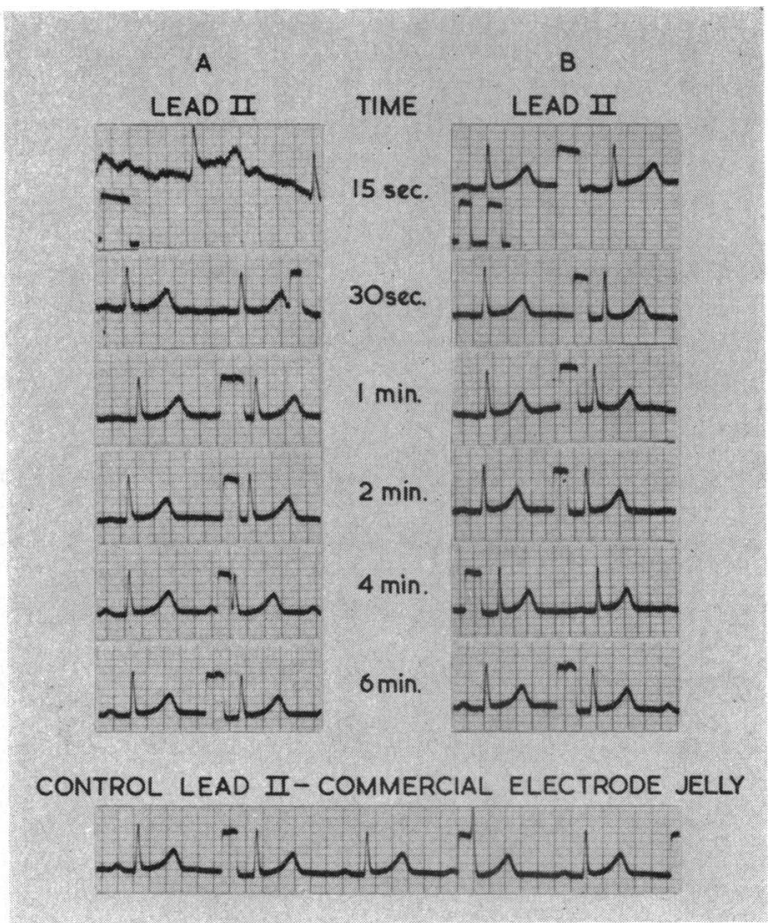

FIG. 4.-Electrocardiograms in a normal girl aged 10 years recorded at timed intervals after applying dry polished electrodes to unprepared skin. (A) Direct electrode-skin contact. (B) After placing one drop of distilled water on the electrode-skin area.

applying the electrodes. A tracing free from interference was obtained in 15 seconds, contrasting with the record at 15 seconds using dry electrodes on unprepared skin in the same subject (Fig. 4). It should be noted, however, that if excess water be used and the electrodes not applied with a firm spreading movement, early records are liable to be distorted or to show interference.

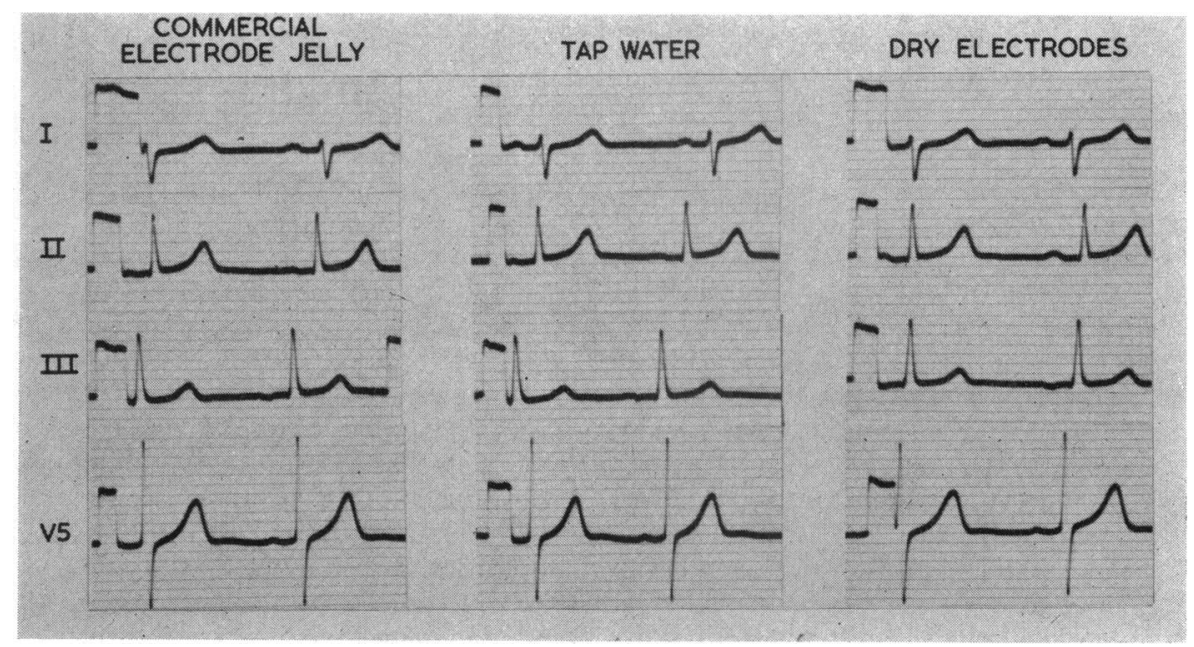

FIG. 5.-Serial electrocardiograms of a healthy girl aged 10 years using: (A) commercial electrode jelly; (B) moistened skin electrode contact area with tap water; (C) dry polished electrodes applied to unprepared skin for 15 minutes. 


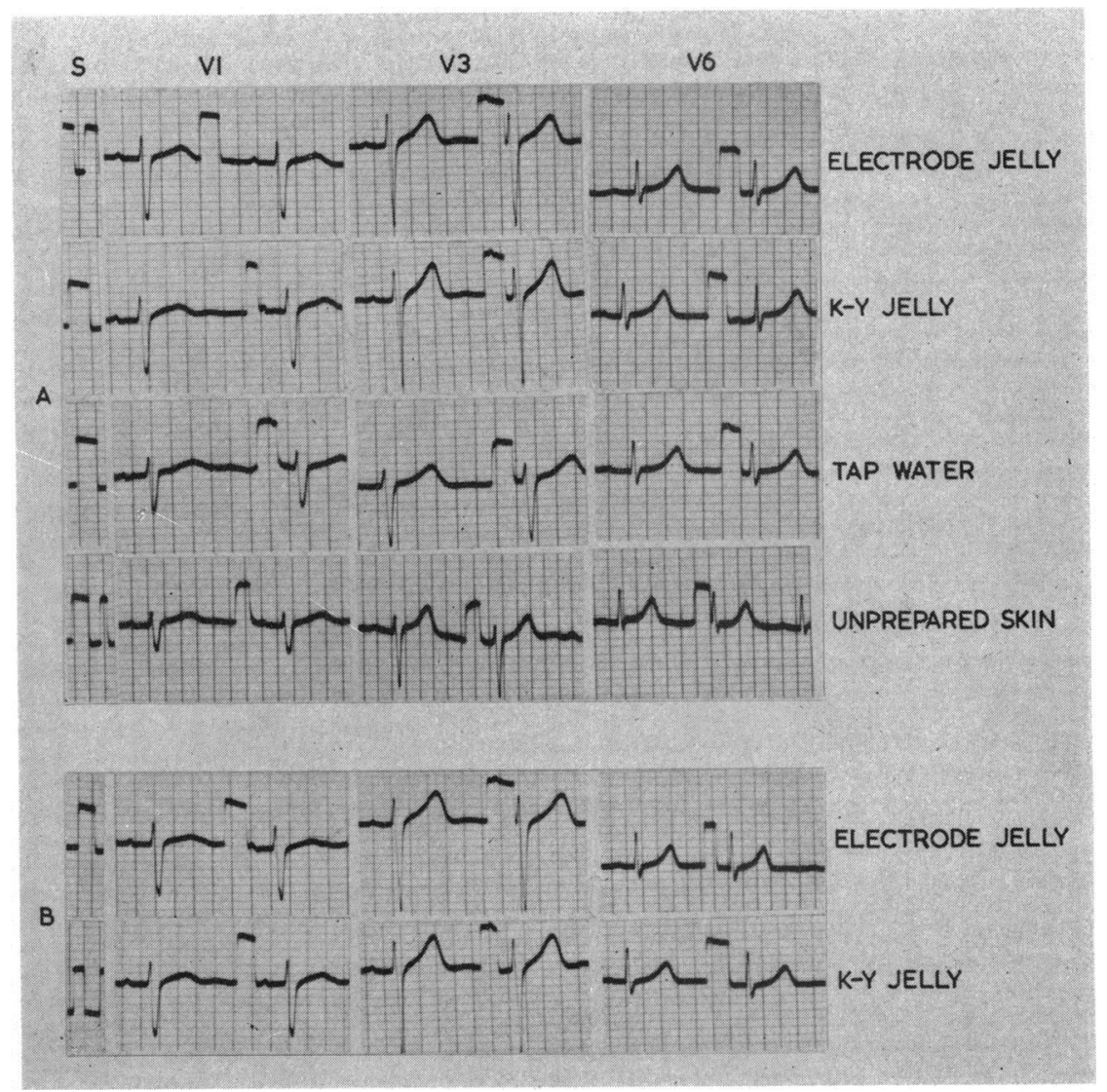

FIG. 6.-Comparative chest electrocardiograms in a normal 10-year-old girl, employing an exploring electrode in (A) of 30 sq. mm. contact area, and in (B) with "point" contact of 1 sq. mm.

Comparative Electrocardiograms using Dry Electrodes and Tap Water. A four lead electrocardiogram was recorded after dry electrodes applied to unprepared skin had been left in position for 15 minutes, and compared with tracings obtained using tap water and commercial electrode jelly. The three methods, tested seriatim in the same subject, gave indistinguishable cardiograms in which the deflection of the standard with the subject in the circuit remained constant (Fig. 5).

Pracordial Electrocardiograms with Limited Chest-Electrode Contact. An experimental suction chest electrode providing an area of skin contact of $30 \mathrm{sq}$. $\mathrm{mm}$. was used for recording the tracings given by dry electrodes, tap water, K-Y, and commercial electrode jelly. The four methods were tested seriatim in the same subject.

$\mathrm{K}-\mathrm{Y}$ and commercial electrode jelly gave strictly comparable tracings; tap water resulted in fall-off in the height of the complexes and standardization with the subject in the circuit. These tendencies were even more apparent when using dry electrodes (Fig. 6A).

"Point-contact" Experiments. A plastic suction electrode containing a free vertical contact of soft iron wire of approximately 1 sq. mm. cross-section was used. Records thus obtained with direct skin contact or tap water were unacceptable owing to gross interference. Records obtained with K-Y Jelly, however, proved the equal of those obtained with commercial electrode jelly (Fig. 6B) and were as acceptable as those yielded by the standard and experimental electrodes of $1 \mathrm{in} .(2.54 \mathrm{~cm}$.) diameter and $30 \mathrm{sq} . \mathrm{mm}$. area contact respectively. 


\section{DISCUSSION OF RESULTS}

The experiments described in this investigation do not uphold the traditional belief that either a high sodium chloride content or abrasives are necessary in order to guarantee an efficient contact at the electrode when using modern single-channel recording equipment with an input impedance of $2 M$ ohm or more. It has been shown that K-Y Jelly, an abrasive-free lubricant of low electrical conductivity, low inorganic ion, and negligible chloride content, and comparable preparations as well as hand cream (Evan Williams), possess the advantages but not the disadvantages of commercial electrode jelly when used for recording electrocardiograms with modern equipment.

The cost of K-Y Jelly is approximately one-third that of commercial electrode jelly and physicians need carry only one preparation for use as an electrode jelly, for lubricating instruments, and for pelvic examination. The preparation is sterile and water-soluble, spreading easily and adhering well. It is harmless to human tissues and will not injure rubber or natural or synthetic textiles. It will not corrode metal instruments and contains neither abrasive nor sodium chloride, in contrast to commercial electrode jelly. Unlike commercial jelly, salt crystals never form in the tube. K-Y Jelly, in the writer's experience, renders shaving of the chest unnecessary even in the most hirsute of men. Its efficient gel system ensures a more effective airlock than does the majority of electrode jellies when recording chest leads.

A few drops of jelly are gently rubbed into the skin with the finger, sufficient being used to ensure a slightly tacky skin surface before applying limb or chest electrodes, which may be conveniently smeared with the finger after preparing the skin. Vigorous preparation of the skin so often required when using string instruments is unnecessary and best avoided. When using suction electrodes on hairy chests, more generous application of K-Y is required. Since the jelly is water-soluble, electrodes are readily cleaned with a damp cloth.

Cardiograms comparable with those obtained with commercial and $\mathrm{K}-\mathrm{Y}$ jellies were recorded when using Lubafax (Burroughs Wellcome). This abrasive-free lubricant of low inorganic ion and negligible chloride content has a specific conductivity of only 0.000233 mhos per ml. compared with a figure for Cambridge jelly nearly 500 times greater. It is evident, therefore, that the Cambridge preparation and comparable electrode contact materials are excessively enriched by sodium chloride. Moreover, specific conductivity measurements take no account of the adjuvant effect which abrasives, such as quartz crystals, or pumice, possess in overcoming skin resistance when preparations containing them are rubbed into the skin (Bell et al., 1939). On the score both of electrolyte and abrasive content, Cambridge electrode jelly and like preparations are overburdened with substances calculated to enhance skin conductivity.

The unexpectedly satisfactory cardiogram recorded by lubricants of low electrical conductivity as well as by a variety of culinary and domestic preparations shows beyond doubt that the high sodium chloride content of commercial electrode jelly is unnecessary for recording acceptable electrocardiograms when using modern recording equipment of high input impedance.

The high quality tracings yielded by simple lubricants of low specific conductivity and by culinary preparations such as marrons glacés, consisting of ground chestnuts, water, and added sugars, caused surprise and gave rise to a doubt on the need of any electrode contact material for the recording of a satisfactory electrocardiogram. It was considered likely that such an hypothesis would be readily disproved by any attempt to record an electrocardiogram using dry polished electrodes applied to the unprepared skin. That such an experiment yielded, after an interval of some four minutes, an electrocardiogram indistinguishable from the control tracing using a commercial electrode jelly was unexpected; but it offered important collateral evidence that the high sodium chloride content of commercial electrode jelly is unnecessary for electrocardiographs of high input impedance.

The experiments when dry polished electrodes were applied to the natural skin or when distilled water was placed between skin and electrodes all yielded faultless electrocardiograms after a variable interval. These experiments suggest, moreover, that any preparation that proves itself efficient as 
a contact material does so more by its capacity to reduce skin resistance through the formation of a thin aqueous conductive film between skin and electrode, than through its inherent inorganic ion content.

The progressive improvement in the electrocardiogram after applying dry polished electrodes to the unprepared skin suggested that condensation of insensible perspiration produced a thin film of moisture containing the natural electrolytes of the sweating skin. When the moisture layer was of sufficient thickness to establish effective skin-electrode contact, resistance was overcome sufficiently to permit the recording of an electrocardiogram free from interference.

The adjuvant effect of a drop of distilled water in permitting the early recording of a faultless cardiogram suggested that the added water not only dissolved skin electrolytes, but also allowed the immediate formation of a thin moisture layer. In the case of dry polished electrodes applied to the natural skin, an effective moisture film with its dissolved electrolytes took time to develop, hence there was a delay of four or more minutes before a satisfactory tracing was obtained.

Since nine widely different preparations each gave equally acceptable electrocardiograms, it is probable that any slightly glutinous aqueous preparation capable of forming and maintaining a thin film of moisture between skin and electrode will serve as an efficient electrode contact material, and that the free inorganic ion content and specific conductivity of a particular preparation is of little practical importance when recording electrocardiograms with modern equipment of high input impedance in subjects with a normal skin.

The experiments described in this study suggest that the area of skin contact afforded by standard suction chest electrodes in common use is more than adequate for recording acceptable electrocardiograms and that the effective area of an electrode must be greatly reduced before there is detectable fall-off in the voltage of the complexes or standardization with the patient in the circuit. Moreover, with progressive reduction of the contact area of the electrode, such decrease in voltage was apparent only when dry electrodes were applied to unprepared skin or when Maxwell's (1957) water method was used; no such fall-off was observed when using K-Y or commercial electrode jelly. The present study, however, confirms Maxwell's observation that no special electrode contact material is necessary for recording acceptable electrocardiograms, and that moistening of skin and electrodes with a damp sponge will suffice when using standard modern equipment. The water method, however, suffers from the disadvantage that an excess of water is likely to give an unsatisfactory or bizarre record, presumably due to undue dilution of natural skin electrolytes. For this reason Maxwell's water method is not advised for routine electrocardiography and a simple abrasivefree lubricant is recommended. The experimental application of dry electrodes to the unprepared skin described in this study is unsatisfactory for routine use not only because of the natural dryness of the skin in some subjects and in most elderly patients, but also on account of the necessary timelag between applying the electrodes and taking the record.

Winsor (1961) has pointed out that electrocardiographs of high input impedance can tolerate high contact resistance provided there is no substantial difference of resistance between any two electrodes. He argues that, in order to obviate wide differences of resistance and thus avoid interference or fallacious electrocardiograms, a standard electrode jelly should be used not only with the string machine and valve amplifying instruments of low impedance, but also with modern high input impedance apparatus. The results of the present study, however, indicate that when using modern instruments of this type the cleanliness of the electrodes and the care with which they are applied are of far greater importance for the recording of acceptable electrocardiograms than is the particular electrode contact material used. A dirty electrode or an electrode sloppily applied is likely to give a tracing which shows interference or is in other ways bizarre, whatever the electrode contact material - be it commercial electrode jelly.

The proposition that careful application of clean electrodes is more important than the electrode contact material when employing modern recording equipment is strongly supported by the fact that polished dry electrodes on unprepared skin repeatedly yielded faultless electrocardiograms that were strictly comparable to the control tracings obtained when using commercial electrode 
jelly. Winsor's argument on the need for standard electrode jelly irrespective of the input impedance of the instrument is difficult to sustain in the light of these findings.

Winsor (1961) also stresses the point that multichannel instruments are more susceptible to high skin resistance than single-channel machines.

Though this argument is not disputed, a double-channel instrument of $4 \mathrm{M}$ ohm input impedance regularly gave acceptable electrocardiograms when using a simple lubricant or hand cream.

The ideal electrode contact material for routine electrocardiography when using single-channel instruments with an input impedance of $2 \mathrm{M} \mathrm{ohm}$ or more is an abrasive-free, dual purpose, watersoluble lubricant containing a small quantity of free electrolytes and a consistency adequate to maintain a thin aqueous film between skin and electrode. K-Y Jelly which has been tested against commercial electrode jelly and proved its equal in performance and superior in cost, convenience, and freedom from corrosive properties, fulfils these requirements. Preparations such as hand cream (Evan Williams) will serve as well, and doubtless other inexpensive bland pharmaceutical and toilet preparations will be found to act just as efficiently as an electrode contact material when using electrocardiographs of high input impedance.

Whatever electrode contact material be used for routine electrocardiography, accurate standardization of the instrument, clean electrodes, and care in applying them nevertheless remain a sine qua non for obtaining acceptable electrocardiograms, and in the case of modern high input impedance machines, to which the claims made in this investigation apply, are more important than the electrode material that is used.

\section{SummaRY AND CONCLUSIONS}

An abrasive-free commercial lubricant jelly of low electrical conductivity, low inorganic ion, and negligible chloride content has been shown, in critical experiments and through the recording of more than 4000 electrocardiograms, to be the equal in electrocardiographic performance and the superior in cost, convenience, and freedom from corrosive properties, to commercial electrode jelly, when using single-channel valve electrocardiographs of input impedance of $2 \mathrm{M}$ ohm or more.

Of nine pharmaceutical and culinary preparations, used as experimental electrode-skin contact material, all gave limb lead cardiograms as acceptable as those obtained when using commercial electrode jelly.

New polished electrodes applied to the natural skin resulted in an electrocardiogram showing gross initial interference that decreased progressively until it gave way to a tracing indistinguishable from a control record obtained with commercial electrode jelly. The placing of a drop of distilled water between polished electrodes and unprepared skin yielded a faultless cardiogram within thirty seconds.

Chest electrocardiograms recorded by dry electrodes applied to the natural skin or to skin moistened with distilled water were as acceptable as those obtained by simple lubricant jelly or commercial electrode jelly.

Water as an electrode contact material, when correctly applied, gives satisfactory electrocardiograms, but is not advised for routine use, and a simple abrasive-free lubricant is recommended.

Experimental chest electrodes of limited skin contact, including point-contact of $1 \mathrm{sq} . \mathrm{mm}$. area, when used with an abrasive-free and chloride-free electrode jelly as electrode contact material yielded an electrocardiogram indistinguishable from that obtained with commercial electrode jelly.

The results of these experiments, which apply only to modern electrocardiographs of high impedance, do not uphold the traditional belief that a high sodium chloride concentration and abrasives are necessary for ensuring efficient contact at the electrode. It is concluded that these substances found in high concentration in commercial electrode jelly are unnecessary for recording acceptable electrocardiograms when using modern recording equipment and that, quite apart from the substantial cost of the preparations containing them, sodium chloride and abrasives may be injurious to plated electrodes or to apparatus. 
I wish to thank Dr. William Evans for his encouragement and help in the preparation of this paper, and Professors J. McMichael, F.R.S., and J. F. Goodwin and Dr. J. H. L. Easton for constructive suggestions.

I thank Miss Y. Cherry, Electrocardiographic Technician to Bedford Hospital, whose initial observation of the efficiency of K-Y Jelly in electrocardiography stimulated this inquiry, for her assistance and for the interest she has shown in this work.

I acknowledge my thanks to Dr. R. U. Russell and Mr. C. R. Watts, Chief Chemists to Johnson and Johnson for their help and co-operation, for carrying out conductivity experiments, and providing information on the chemical composition and the manufacture of K-Y Jelly.

To Mr. A. N. Provost, General Manager European Semi-Conductor Group of Texas Instrument Company, I am indebted for helpful criticism and expert technical advice and for his suggesting further experiments on electrode jellies, and to Dr. Shirley Smith, Mr. L. Goulding, F.S.C.T., Chief Technician to the Cardiac Department, University Coliege Hospital, and Mr. E. G. Short, Electrocardiographic Technician, Chester Royal Infirmary, for advice on technical and other matters.

\section{REFERENCES}

Browne, M. K. (1957). Preparation of skin for electrocardiography. Brit. med. J., 2, 1238.

Bell, G. H., Knox, J. A. C., and Small, A. J. (1939). Electrocardiograph electrolytes. Brit. Heart J., 1, 229.

Boas, E. P. (1928). The cardiotachometer: an instrument to count the totality of heart beats over long periods of time. Arch. intern. Med., 41, 403.

Cohn, A. E. (1920). A new electrode for use in clinical electrocardiography. Arch. intern. Med., 26, 105.

Hartridge, H. (1931). Methods of reducing the errors in electrical testing due to variations in skin resistance. Brit. J. Radiol., 4, 652.

James, W. B., and Williams, H. B. (1910). The electrocardiogram in clinical medicine. I. The string galvanometer and the electrocardiogram in health. Amer. J. med. Sci., 140, 408.

Jenks, J. L., and Graybiel, A. (1935). A new and simple method of avoiding high resistance and overshooting in taking standardized electrocardiograms. Amer. Heart J., 10, 693.

Maxwell, J. (1957). Preparation of skin for electrocardiography. Brit. med. J., 2, 942.

(1958). Preparation of skin for electrocardiography. Brit. med. J., 1, 41.

Russell, H. B. (1935). The use of "Cambridge electrode jelly". Lancet, 2, 1172.

Winsor, T. (1961). Skin resistance and abnormal electrocardiography. General Pract., $24,9$. 